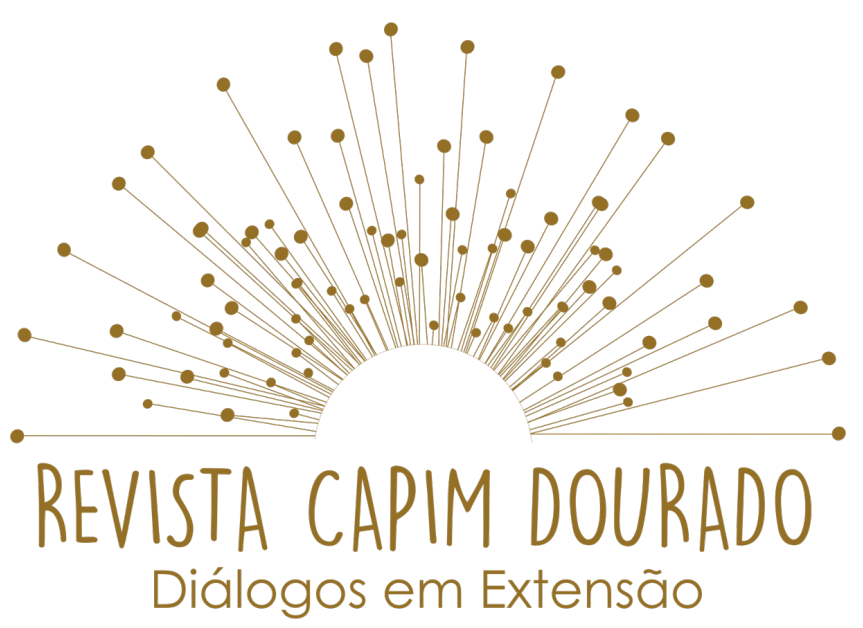

ISSN n² 2595-7341

Vol. 3, n. 1, Janeiro-Abril, 2020

DOI: http://dx.doi.org/10.20873/uft.2595-7341.2020v3n1p151

\title{
A UNIVERSIDADE ABERTA À COMUNIDADE: CONHECENDO O COMPLEXO DE LABORATÓRIOS DA SAÚDE - CAMPUS DE PALMAS
}

THE UNIVERSITY OPEN TO THE COMMUNITY: KNOWING THE COMPLEX OF HEALTH LABORATORIES - CAMPUS DE PALMAS

LA UNIVERSIDAD ABIERTA A LA COMUNIDAD: CONOCIENDO EL COMPLEJO DE LABORATORIOS DE SALUD - CAMPUS DE PALMAS

\author{
Adriane Trindade Gonçalves de Lima ${ }^{1}$ \\ Kércio Jeaneryson Nogueira de Sousa Leite ${ }^{2}$ \\ Aline Queiroz Fernandes, 3 \\ Andressa Diúlia Dantas da Silva ${ }^{4}$ \\ Ediana Vasconcelos Silva ${ }^{5}$
}

\section{RESUMO}

O presente trabalho apresenta discussões acerca da vivência prática de visitas técnicas realizadas pela demanda externa de estudantes de colônia de férias, ensino fundamental, médio e superior ao complexo de laboratórios da saúde da

1 Acadêmica de Nutrição, Universidade Federal do Tocantins (UFT), Palmas, Tocantins, adrianetglima@mail.uft.edu.br.

2 Acadêmico de Enfermagem, Faculdade de Palmas (FAPAL), Palmas, Tocantins, kerciojeaneryson@hotmail.com.

3 Acadêmica de Nutrição, Universidade Federal do Tocantins (UFT), Palmas, Tocantins, alineqf@mail.uft.edu.br.

4 Acadêmica de Nutrição, Universidade Federal do Tocantins (UFT), Palmas, Tocantins, andressa.diulia10@gmail.com.

5 Coordenadora dos laboratórios da saúde, Universidade Federal do Tocantins (UFT), Palmas, Tocantins, edianavasconcelos@mail.uft.edu.br. 


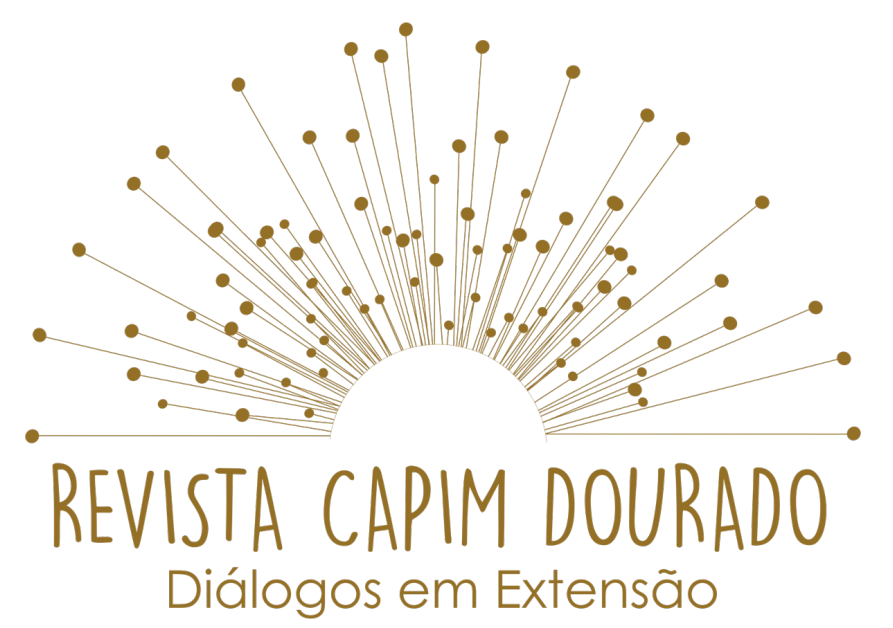

ISSN n² 2595-7341

Vol. 3, n. 1, Janeiro-Abril, 2020

DOI: http://dx.doi.org/10.20873/uft.2595-7341.2020v3n1p151

Universidade Federal do Tocantins (UFT). Ao proporcionar visitas técnicas se estreita as relações entre a comunidade e a universidade ampliando as oportunidades para obtenção de conhecimentos com benefícios mútuos, que é o objetivo do trabalho. Foram confeccionados materiais para apresentação dos métodos de ingresso ao ensino superior, assim como apresentar os cursos ofertados na UFT para tentar despertar o interesse por esta instituição, além disto, a equipe do projeto apresenta os conteúdos nos laboratórios que as escolas visam aprofundamento prático. Dessa forma, o projeto teve uma ótima aceitação pública, tanto que, foram recebidas escolas de todo o estado do Tocantins, desde os municípios circunvizinhos aos periféricos, com uma demanda média de três visitas por mês. As experiências vivenciadas pelos extensionistas e colaboradores do projeto além de despertar o interesse pela docência favorece o vínculo com a comunidade externa que é de suma importância para o futuro profissional.

PALAVRAS-CHAVE: Universidade. Visitas. Ciências Básica. Comunidade. Ensino Médio.

\section{ABSTRACT}

The present work presents discussions about the practical experience of technical visits made by the external demand of students from summer camp, elementary, middle and higher education to the complex of health laboratories at the Federal University of Tocantins (UFT). By providing technical visits, the relationship between the community and the university is strengthened, expanding the opportunities for obtaining knowledge with mutual benefits, which is the objective of the work. Materials were prepared to present the methods of entering higher education, as well as presenting the courses offered at UFT to try to arouse interest in this institution. In addition, the project team presents the contents in the laboratories that schools aim at furthering in practice. Thus, the project had a great public acceptance, so much so that schools were received from all over the state of Tocantins, from the surrounding municipalities to the 


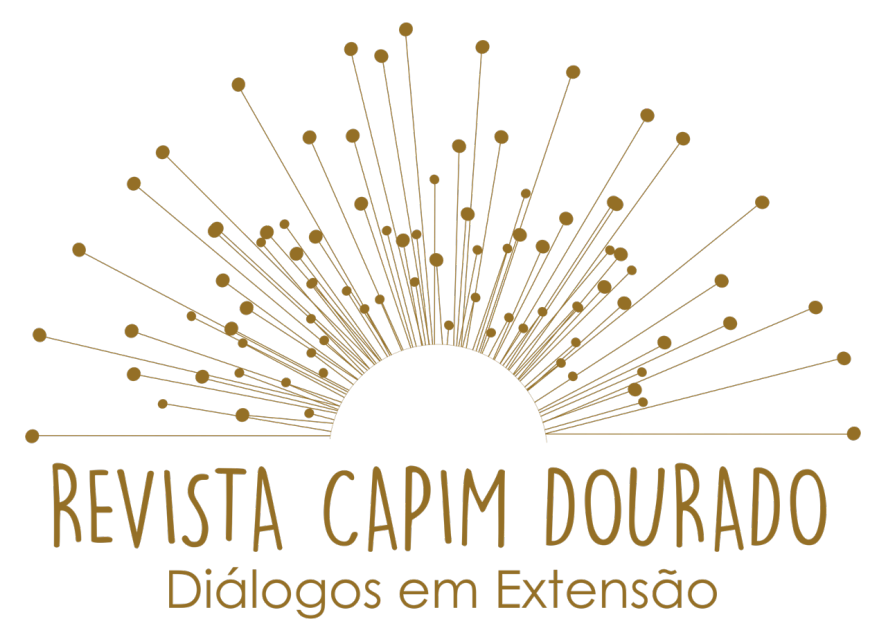

ISSN n² 2595-7341

Vol. 3, n. 1, Janeiro-Abril, 2020

DOI: http://dx.doi.org/10.20873/uft.2595-7341.2020v3n1p151

peripheries, with an average demand of three visits per month. The experiences of extension workers and project collaborators, in addition to arousing interest in teaching, favor the bond with the external community, which is of paramount importance for the professional future.

KEYWORDS: University. Visits. Basic Sciences. Community. High school.

\section{RESUMEN}

El presente trabajo presenta discusiones sobre la experiencia práctica de visitas técnicas realizadas por la demanda externa de estudiantes de campamento de verano, educación básica, media y superior al complejo de laboratorios de salud de la Universidad Federal de Tocantins (UFT). Al brindar visitas técnicas, se fortalece la relación entre la comunidad y la universidad, ampliando las oportunidades de obtención de conocimiento con beneficio mutuo, que es el objetivo del trabajo. Se prepararon materiales para presentar los métodos de ingreso a la educación superior, así como presentar los cursos que se ofrecen en la UFT para intentar despertar el interés en esta institución, además, el equipo del proyecto presenta los contenidos en los laboratorios que las escuelas pretenden promover en la práctica. Así, el proyecto tuvo una gran aceptación pública, tanto que se recibieron escuelas de todo el estado de Tocantins, de los municipios aledaños a las periferias, con una demanda promedio de tres visitas por mes. Las experiencias de los extensionistas y colaboradores del proyecto, además de despertar el interés por la docencia, favorecen el vínculo con la comunidad externa, que es de suma importancia para el futuro profesional.

PALABRAS CLAVE: Universidad. Visitas. Ciencias Basicas. Comunidad. Escuela secundaria.

Recebido em: 01.10.2019. Aceito em: 09.10.2019. Publicado em: 01.01.2020. 


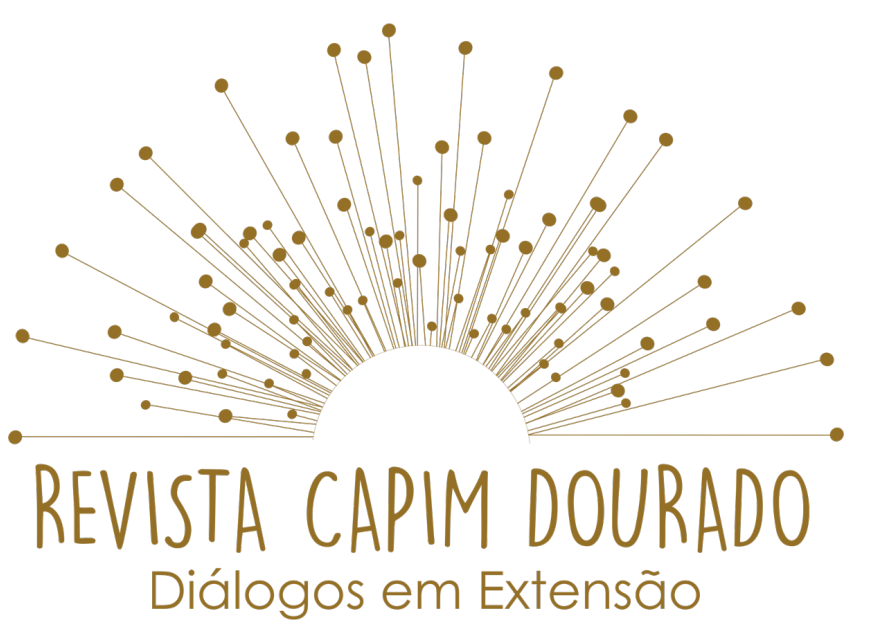

ISSN n² 2595-7341

Vol. 3, n. 1, Janeiro-Abril, 2020

DOI: http://dx.doi.org/10.20873/uft.2595-7341.2020v3n1p151

\section{INTRODUÇÃO}

O projeto "A Universidade aberta à comunidade: conhecendo o complexo de laboratórios da saúde" é um projeto de extensão criado na Universidade Federal do Tocantins - Campus Palmas com o intuito de atender a demanda da comunidade para visitas técnicas que são solicitadas à coordenação dos Laboratórios da Saúde da UFT.

As Universidades brasileiras têm a extensão como um dos eixos fundamentais do tripé formado pelo ensino, pesquisa e extensão. Essa possui como base, a troca de conhecimentos entre comunidade acadêmica e sociedade, com atividades que permitem a aproximação dos estudantes ao ambiente científico, proporcionando integração entre os mais diversos agentes do processo ensino-aprendizagem (BORGES, 2010).

Sob o ponto de vista de Ausubel, 2000, a ideia citada anteriormente pode contribuir para a ancoragem de conhecimentos com organizadores prévios que os estudantes trazem consigo, proporcionando uma visão crítica e evitando a aprendizagem mecânica.

A componente curricular que contempla as ciências básicas, está presente tanto no Ensino Superior quanto no Ensino Básico, dentro de suas respectivas habilidades e competências. Por isso esse projeto tem como objetivo geral integrar esses dois níveis de formação, básica e superior, e, além disso, aproximar docentes, técnicos e discentes da UFT com a comunidade para que possam interagir entre si e complementar saberes e despertar o interesse científico. 


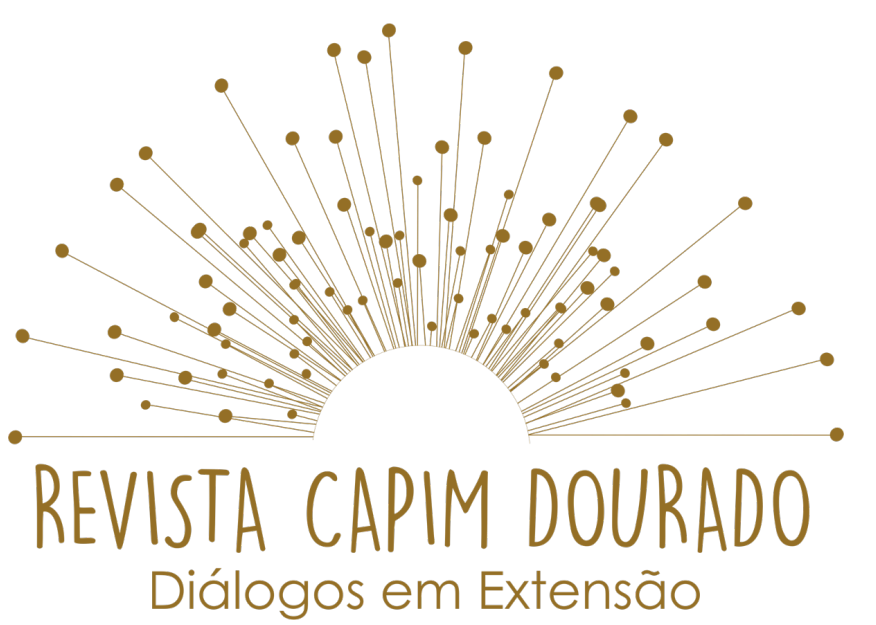

ISSN n² 2595-7341

Vol. 3, n. 1, Janeiro-Abril, 2020

DOI: http://dx.doi.org/10.20873/uft.2595-7341.2020v3n1p151

\section{METODOLOGIA}

O projeto "A Universidade aberta à comunidade: conhecendo o complexo de laboratórios da saúde" - Campus de Palmas, surgiu a partir de outro projeto denominado "Anatomia para todos: a Universidade aberta à comunidade", ao observar que nossos objetivos não estavam sendo alcançados apenas com a visita ao laboratório de anatomia humana, havendo a necessidade de aumentar as vivências práticas para melhor atender as solicitações dos visitantes.

As visitas ao laboratório de anatomia se iniciaram no ano de 2010, porém só foram oficializadas no formato de projeto de extensão no ano de 2017. A partir disso, foram realizadas reuniões para organizar o recebimento dos alunos, com o intuito de apresentar a maior quantidade de laboratórios possíveis. Ficou definido que o grupo de maior importância para as visitas seria os alunos do ensino médio, tendo em vista que a oportunidade de conhecer laboratórios na área da saúde pode influenciar positivamente suas escolhas por uma graduação.

Para melhor atender as demandas do projeto foram desenvolvidos materiais visuais pela bolsista PIBEX e alunos extensionistas voluntários, como slides interativos e panfletos, com o objetivo de apresentar a Universidade Federal do Tocantins - UFT e todos os cursos oferecidos por ela, o ENEM e suas especificidades, programas para ingressar no ensino superior e o sistemas de cotas. Quanto aos panfletos, estes são entregues durante a visita ao público, contendo as informações citadas na apresentação feita pela equipe do projeto.

Para captar o público-alvo do projeto, foram confeccionados panfletos para auxiliar os convites feitos às instituições. Algumas escolas foram visitadas e 


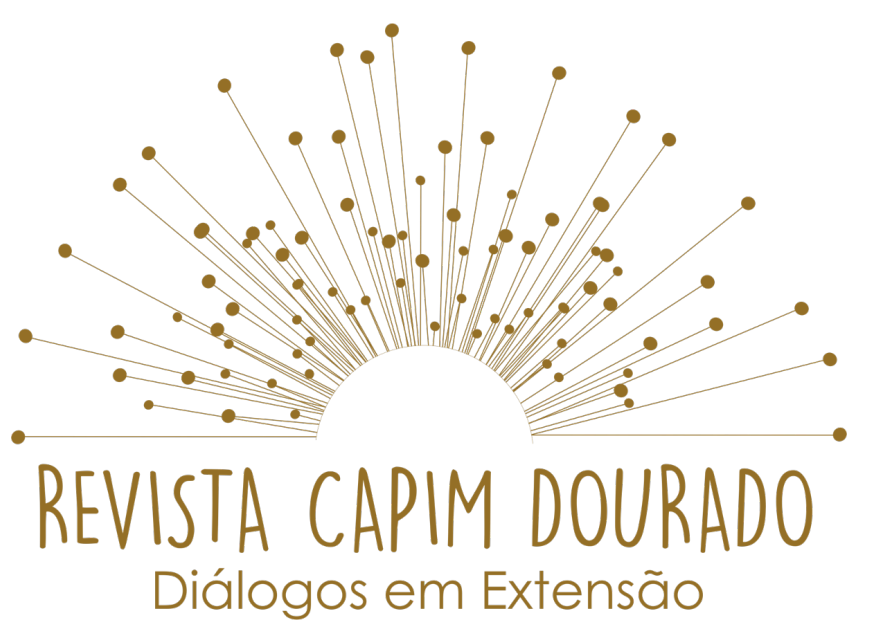

ISSN n² 2595-7341

Vol. 3, n. 1, Janeiro-Abril, 2020

DOI: http://dx.doi.org/10.20873/uft.2595-7341.2020v3n1p151

convidadas a participar do projeto, enquanto outras eram convidadas por telefone e e-mail.

As visitas são realizadas aos laboratórios de anatomia, bioquímica, histologia, enfermaria modelo, microbiologia, parasitologia, técnicas cirúrgicas, entre outros, de acordo com a disponibilidade. Durante todo o percurso, os técnicos de laboratório, discentes voluntários, bolsista, monitores e professores realizam o acompanhamento, promovendo uma interação complementar dos saberes, podendo despertar o interesse científico.

Como forma de avaliação do trabalho feito no projeto é realizada a captação de feedback's por meio oral e escrito.

\section{FUNDAMENTAÇÃO TEÓRICA}

As atividades práticas no ensino de Ciências podem ser vistas sob o ponto de vista da teoria construtivista de Piaget, a partir da qual o conhecimento pode ser construído com base em ações concretas ou mentais. Conforme Oliveira, 2010, entre as atividades experimentais ou práticas, podemos citar as atividades de demonstração, verificação e investigação, como maneiras de aproximar o conhecimento teórico da realidade e do cotidiano daquele que o constrói. Sendo assim, tais atividades constituem-se como forma de aproximar os estudantes do ambiente científico, proporcionar integração entre os mais diversos agentes do processo ensino-aprendizagem, melhorar a capacidade de trabalho em grupo e de avaliação crítica. 


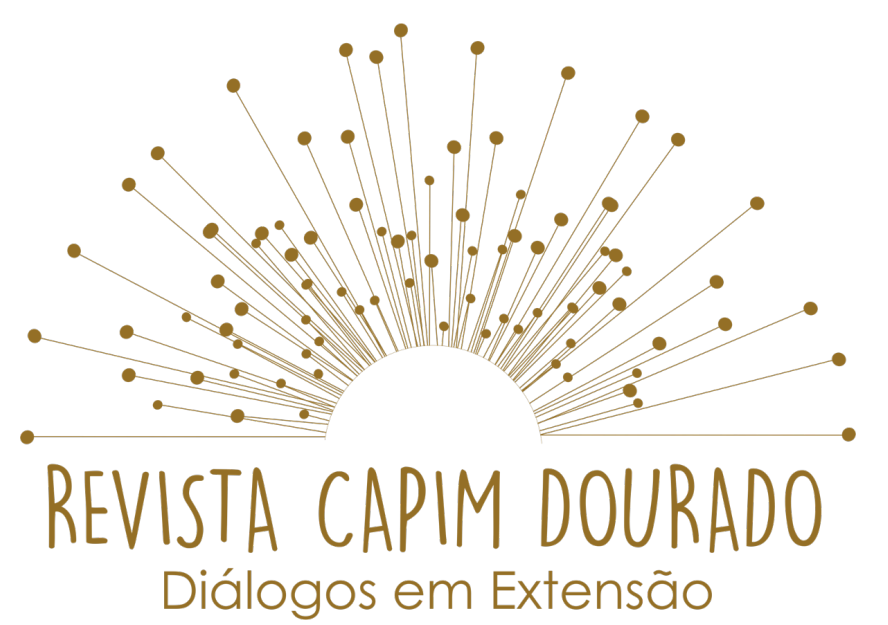

ISSN n² 2595-7341

Vol. 3, n. 1, Janeiro-Abril, 2020

DOI: http://dx.doi.org/10.20873/uft.2595-7341.2020v3n1p151

As ciências básicas contempladas na educação básica nas escolas da rede de ensino público ou privado dificilmente possuem infraestrutura, tais como laboratórios equipados para aulas práticas, o que pode prejudicar o processo de ensino-aprendizagem (Mclachlan \& Patten, 2006). Para suprir essas deficiências, há várias alternativas como vídeos, jogos, modelos interativos, que podem ser usadas como recursos didáticos no ensino. Outro meio de reforçar esse processo é aproximar as escolas das universidades, por meio dos projetos de pesquisa.

Borges \& Fontoura, 2010, apontam a ideia de circularidade indicando a conexão entre as duas fontes produtoras do saber (escola e universidade). Para Tabolka \& Grotto, 2012, as atividades de pesquisa permitem a possibilidade de comunicação/divulgação dos conhecimentos produzidos nas universidades atingindo dessa maneira um segmento maior da sociedade.

\section{RESULTADOS FINAIS}

Este projeto de extensão pôde proporcionar a alunos de escolas públicas de cidades do interior do Tocantins como Paraíso, Porto Nacional, Tocantínia, Formoso do Araguaia e Pindorama uma experiência ímpar com a Universidade pública. Foi possível receber cerca de três ou mais turmas por mês e o gratificante foi ver que o projeto não atingiu apenas alunos do ensino médio, atendendo a demanda com o intuito de agregar o conhecimento de forma geral, recebendo alunos do ensino fundamental, crianças da colônia de férias da UFT que desde bem pequenos demonstram grande interesse pelo estudo macroscópico do corpo humano, fizemos parceria com uma professora de Anatomia, sendo 


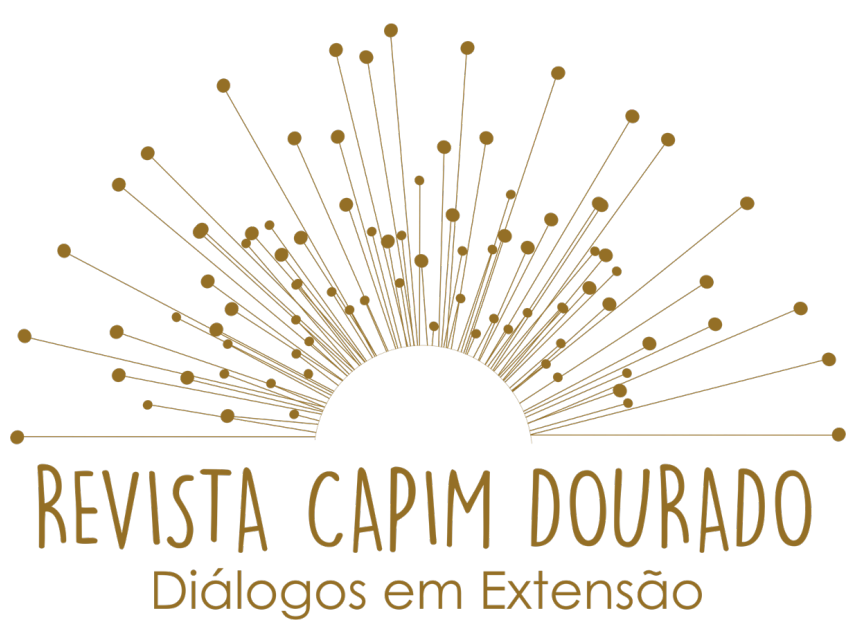

ISSN n² 2595-7341

Vol. 3, n. 1, Janeiro-Abril, 2020

DOI: http://dx.doi.org/10.20873/uft.2595-7341.2020v3n1p151

realizados intensivos práticos para alunos de enfermagem de faculdades particulares que não tem em seus laboratórios um grande acervo como o da UFT e durante esses intensivos foram atendidos muitos alunos, demostrando que 0 projeto é de suma importância para a complementação de conhecimentos. Além disto, atendemos a todas as solicitações das escolas.

A organização para receber as visitas, resultou em conteúdos que foram apresentados nos laboratórios, sendo estes moldados em materiais visuais, gráficos e práticos de acordo com a demanda do público visitante. As experiências vividas pelos colaboradores do projeto possibilitaram a descoberta de interesses à área da docência, e a interação entre eles e o público proporcionou o exercício do vínculo entre ensino, pesquisa e extensão (MOITA, 2009).

\section{CONSIDERAÇÕES FINAIS}

Este projeto trabalha as relações de circularidades de saberes exercida no cotidiano existente entre a escola e a Universidade, sendo estas duas fontes produtoras de saber, o que está de acordo com a abordagem de LUDKE (2005).

Sendo assim, o tripé Ensino, Pesquisa e Extensão esteve presente durante a execução do projeto, pois ao atender a comunidade os discentes reforçam os conhecimentos previamente adquiridos, pois, desenvolvem e praticam a oratória, além de que, fortalecem as relações com a comunidade externa. Além disso, no decorrer do projeto, firmou-se uma parceria com o projeto de extensão, "Preparação de peças anatômicas para implementação do Museu de Morfologia 


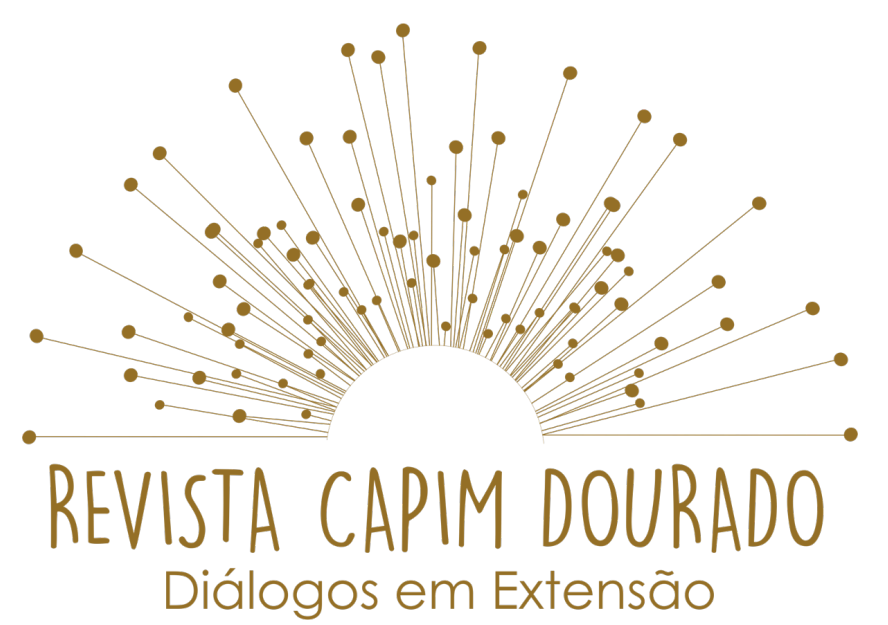

ISSN n² 2595-7341

Vol. 3, n. 1, Janeiro-Abril, 2020

DOI: http://dx.doi.org/10.20873/uft.2595-7341.2020v3n1p151

da Universidade Federal do Tocantins", e este vinculo proporciona aos alunos uma interação ainda maior entre a teoria e a prática que futuramente proporcionará novos espaços para visitação.

Objetivando o trabalho de pesquisa científica, este projeto visa produzir artigos sobre as abordagens coletivas realizadas e vivenciadas durante as visitas aos laboratórios da saúde da Universidade Federal do Tocantins - Campus Palmas. Sendo assim, far-se-á necessário a continuidade deste projeto para que visitas continuem favorecendo aos estudantes a oportunidade de conhecer 0 complexo de laboratórios da saúde e ampliando os seus conhecimentos com a abertura da universidade.

\section{REFERÊNCIAS}

BORGES, L.; FONTOURA, H. Diálogos entre a escola de educação básica e a universidade: a circularidade de saberes na formação docente. Intermeio. 16:32, 143-156. 2010.

AUSUBEL, D.P. The acquisition and retention of knowledge. Dordrecht: Kluwer Academic Publishers. 2000.

OLIVEIRA, J. R. S. Contribuições e abordagens das atividades experimentais no ensino de ciências: reunindo elementos para a prática docente. Acta Scientiae. 12:1, 139-153. 2010.

MCLACHLAN, JC; PATTEN, D. Anatomy teaching: ghosts of the past, a present and future. Med. Educ. 40:243-253. 2006. 


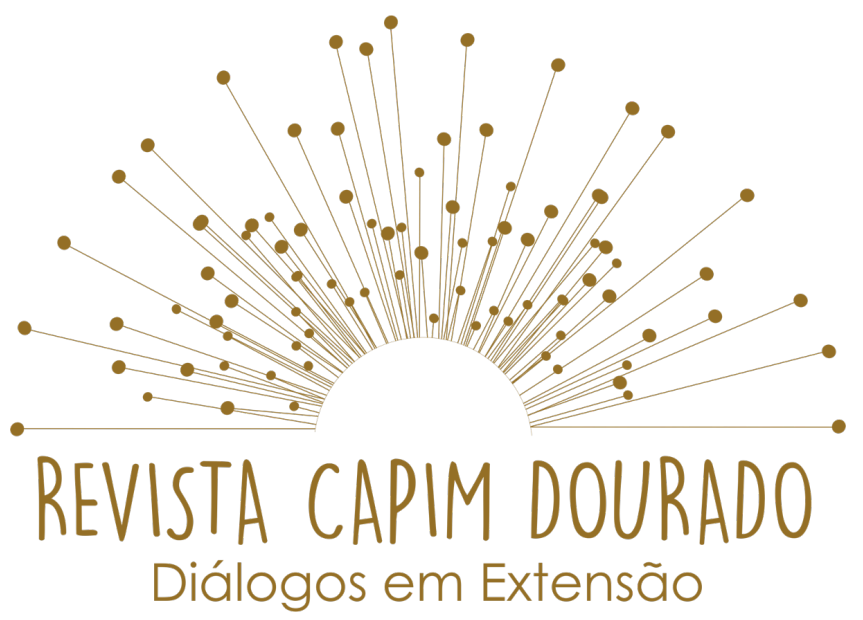

ISSN n² 2595-7341

Vol. 3, n. 1, Janeiro-Abril, 2020

DOI: http://dx.doi.org/10.20873/uft.2595-7341.2020v3n1p151

TABOLKA, C. C; GROTTO, E. M. B; Universidade e Escola: Diferentes culturas que se encontram em diferentes momentos. 14-18, 2012.

MOITA, F. Ensino-pesquisa-extensão: um exercício de indissociabilidade na pósgraduação. Revista Brasileira de Educação v. 14 n. 41. 2009.

LUDK, M. Aproximando Universidade e Educação Básica pela Pesquisa no Mestrado. Projeto de Pesquisa - Departamento de Educação: PUC-Rio, 2005. 